\title{
Genetic Influence on Accessory Navicular Bone in the Foot: A Korean Twin and Family Study
}

\author{
In Yae Cheong, ${ }^{1}$ Hyo Jeong Kang, ${ }^{1,2}$ Hyeonyoung Ko, ${ }^{3}$ Joohon Sung, ${ }^{4,5}$ Yun-Mi Song, ${ }^{3}$ \\ and Ji Hye Hwang ${ }^{1}$ \\ ${ }^{1}$ Department of Physical and Rehabilitation Medicine, Samsung Medical Center, Sungkyunkwan University School of \\ Medicine, Seoul, South Korea \\ ${ }^{2}$ Department of Physical Therapy, Graduate School, Sahmyook University, Seoul, South Korea \\ ${ }^{3}$ Department of Family Medicine, Samsung Medical Center, Sungkyunkwan University School of Medicine, Seoul, South \\ Korea \\ ${ }^{4}$ Department of Epidemiology, School of Public Health, Seoul National University, Seoul, South Korea \\ 5 Institute of Health and Environment, Seoul National University, Seoul, South Korea
}

\begin{abstract}
An accessory navicular bone (AN) is the most common accessory ossicle in the foot. The presence of an AN bone can trigger various foot problems, such as posterior tibial tendon pathology, flattening of the medial longitudinal arch, and medial foot pain. Despite the clinical influence of presence of an AN in foot disease, the research regarding its inheritance is still insufficient. A total of 135 pairs of monozygotic (MZ) twins, 25 pairs of dizygotic (DZ) twins, and 676 singletons from families were enrolled in order to estimate genetic influences on AN. After confirmation of zygosity and family relationship with a tandem repeat marker kit and questionnaires, the presence and type of the AN was classified through bilateral feet radiographic examination. The heritability of an AN was estimated using quantitative genetic analysis based on a variance decomposition model considering various types of family relationships: father-offspring pair, mother-offspring pair, and pooled DZ twin and sibling pairs. As a result, approximately $40.96 \%$ of the participants in this study had an AN in either foot, with type II being the most common type. The heritability for the presence of any type of an AN in any foot was estimated as $0.88(95 \% \mathrm{Cl}[0.82,0.94])$ after adjusting for age and sex. Specifically, type II AN showed the highest heritability of $0.82(95 \% \mathrm{Cl}[0.71-0.93])$. The high heritability of an AN found in this large twin and family study suggests that an AN is determined by the substantial influence of genetic factor.
\end{abstract}

Keywords: accessory navicular bone, foot, twin, heritability

The accessory navicular bone (AN) is an extra bone located medial to the tuberosity of the tarsal navicular bone (Offenbecker \& Case, 2012). It is an anatomic variant that develops as a result of an incompletely fused secondary ossification center of the navicular tuberosity. The AN is classified into three types according to its shape and connection to the navicular bone (Figure 1). Type I is a small sesamoid bone embedded in the posterior tibial tendon, type II is a heart-shaped or triangular ossicle that is connected to the navicular by synchondrosis, and type III is an enlarged medial horn of the navicular after fusion of the extra bone (Vaughan \& Singh, 2014).

An $\mathrm{AN}$ is the most common ossicle in the foot (Coskun et al., 2009), with an overall incidence of $4-21 \%$ in the general population (Coskun et al., 2009; Miller et al., 1995). An AN may be symptomatic, causing pain in the medial foot and other resultant problems, including rare cases of fracture (Mikami \& Azuma, 1978). The accessory ossicle in the navicular may cause the posterior tibial tendon to insert either directly into the AN or become displaced inward and upward (Kiter et al., 1999; Vaughan \& Singh, 2014), which may lead to tendon insufficiency followed by decreased dynamic Stability and possibly progressive

RECEIVED 5 December 2016; ACCEPTEd 15 March 2017. First published online 10 April 2017.

AdDress For Correspondence: Ji Hye Hwang MD, PhD, Department of Physical and Rehabilitation Medicine, Samsung Medical Center, Sungkyunkwan University School of Medicine, 81 Irwon-ro, Gangnam-gu, Seoul 06351, South Korea. E-mail: hwanglee@skku.edu 


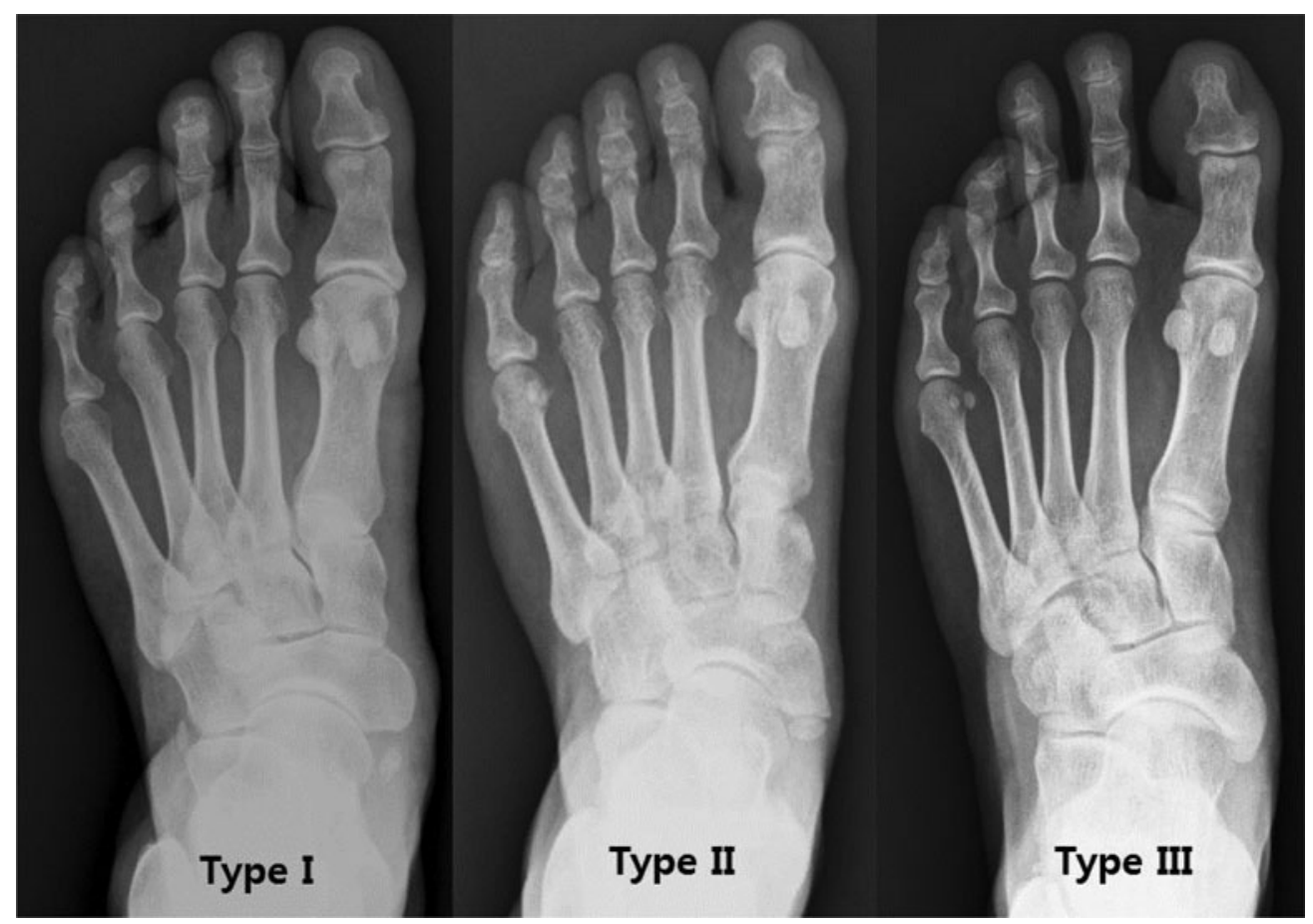

\section{FIGURE 1}

The foot radiographs of three different types of accessory navicular bone.

flattening of the longitudinal arch of the foot (Chen et al., 1997; Keles Coskun et al., 2009; Offenbecker \& Case, 2012; Vaughan \& Singh, 2014). The presence of an AN is also associated with medial foot pain, which occurs at its interface with the navicular tuberosity (Offenbecker \& Case, 2012), also called painful navicular syndrome (Bernaerts et al., 2004), and it may lead to degenerative changes and restricted range of motion due to overuse or trauma (Huang et al., 2014). The symptomatic AN can be managed with treatment ranging from conservative methods, such as activity modification, comfortable shoes with orthosis, and physical therapy to eventual surgical intervention in refractory cases (Seehausen et al., 2016; Vaughan \& Singh, 2014). This aspect of an AN in relation to posterior tibial tendon pathology, flat foot, and a possible cause of medial foot pain has generated growing interest among clinicians.

The increasing clinical role of an AN in foot pathology has resulted in increased attention to its inheritance, with respect to whether it is passed down from generation to generation. Nevertheless, only a few studies have documented the inheritance of an AN by examining the pedigree of the families (Dobbs \& Walton, 2004; Kiter et al., 2000), and evaluation of genetic influence on an AN in a large sample has not yet been done.

This study aimed to estimate the heritability of an AN using data from a large-size study of twins and family that provide various types of family relationships.

\section{Materials and Methods}

\section{Subjects}

The study included a total of 996 Korean adults composed of twins of the same gender and their first-degree relatives from the participants enrolled in the Healthy Twin Study from May 2008 to April 2010. They consisted of $135 \mathrm{MZ}$ twin pairs, $25 \mathrm{DZ}$ pairs, and 676 singleton family members of the twins. From them, 280 father-offspring pairs, 477 mother-offspring pairs, and 266 pooled DZ and sibling pairs were identified in addition to the twin pairs.

The Healthy Twin Study was a cohort study initiated in 2005 in order to investigate genetic and environmental aspects of complex diseases. Details of the study design and protocols have been previously published (Gombojav et al., 2013). Study participants were recruited through a nationwide advertisement and a mail-out to some members of the Korean twin-family register whose contact information was available. All participants of the healthy twin study underwent full medical examinations and completed detailed questionnaires asking about lifestyle and epidemiologic characteristics, after providing written informed consent. The operation or any intervention of the feet were identified and excluded. The persons who participated in the Healthy Twin Study between May 2008 and April 2010, additionally underwent physical examinations and radiographic measurements of feet in order to assess the presence of an AN and its type. 
TABLE 1

Characteristics of the Study Participants

\begin{tabular}{|c|c|c|c|}
\hline & All $(n=996)$ & Women $(n=604)$ & Men $(n=392)$ \\
\hline Age, year & $43.99 \pm 13.26$ & $44.11 \pm 12.78$ & $43.82 \pm 13.99$ \\
\hline Body mass index, $\mathrm{kg} / \mathrm{m}^{2}$ & $23.87 \pm 3.16$ & $23.36 \pm 3.21$ & $24.64 \pm 2.91$ \\
\hline Twins & $320(32.13)$ & $210(34.77)$ & $110(28.06)$ \\
\hline Monozygotic & $270(27.11)$ & $184(30.46)$ & $86(21.94)$ \\
\hline Dizygotic & $50(5.02)$ & $26(4.30)$ & $24(6.12)$ \\
\hline Overall prevalence of an accessary navicular bone ${ }^{a}$ & $408(40.96)$ & $257(42.55)$ & $151(38.52)$ \\
\hline
\end{tabular}

Note: Data were presented as number (percentage) for categorical variables or mean \pm standard deviation for continuous variables. aThe presence of any type of an accessory navicular bone in the right foot or the left foot.

The study was approved by the Institutional Review Board at the Samsung Medical Center (IRB file No.: SMC2005-08-113)

\section{Radiographic Assessment}

Foot X-rays with an antero-posterior and lateral weightbearing view were taken from all study participants. Two physicians well-acquainted with the types of ANs determined the presence and the type of an AN based on the interpretation of foot X-rays according to Coughlin's classification (Coughlin, 2006). Inter-rater reliability between these two physicians was quantified using Cohen's kappa coefficient and it showed high reliability for any type of the $\mathrm{AN}$, ranging from 0.74 to 0.95 (Table S1). The round- or oval-shaped small accessory ossicle without connection to the navicular bone was determined as Type I. Type II was defined when a triangular or heart-shaped bone was present with its base connected to the talar process by an acute angle or when it was situated inferiorly to the navicular bone. Last, when the AN was united to the navicular by a bony ridge, forming cornuate navicular bone, it was classified as Type III (Coughlin, 2006; Huang et al., 2014).

\section{Zygosity and Family Relationship Ascertainment}

Sixteen short tandem repeat marker kits (PerkinElmer's AmpFlSTR ${ }^{\circledR}$, Norwalk, CT) were used to determine the zygosity of twins and to ascertain the family relationship of the other participants. A zygosity questionnaire developed by the research team, which showed a predictive value of more than 98\% for MZ twins (Song et al., 2010), was also used as a supplementary mean for determining zygosity.

\section{Statistical Analysis}

Correlations within each various intrafamilial pair for the presence and classification of an $\mathrm{AN}$ were calculated using Cohen's kappa coefficient. The agreement for the presence and classification of an AN between the left foot and the right foot was evaluated using the chi-square test. A $p$ value less than .05 was considered statistically significant. Descriptive statistics, calculation of the kappa coefficients, and a chi-square test were performed using statistical software (SAS software 9.4; SAS Inc., Cary, NC).

Heritability was calculated as the proportion of the total phenotypic variance explained by additive genetic effects in a statistical genetic analysis software package (Sequential Oligogenic Linkage Analysis Routines, Eclipse version 7.6.4; Allison et al., 1999; Almasy \& Blangero, 1998). Total phenotypic variation in an $\mathrm{AN}$ after adjusting for linear and non-linear effects of age, sex, and interactions between age and sex was partitioned into additive genetic $\left(б \mathrm{a}^{2}\right)$, shared environmental component within a family $\left(\sigma c^{2}\right)$, and individual specific unique environmental component $\left(\sigma \mathrm{e}^{2}\right)$, according to the variance decomposition model. The key assumption of this model was that the effects of environmental factors are common to the members of a family and that the three factors $\left(\sigma a^{2}, \sigma c^{2}\right.$, and $\left.\sigma e^{2}\right)$ have independent and additive effects on the trait variance, with the total residual variance being the sum of the additive and individual specific variance components $\left(\sigma \mathrm{p}^{2}=\sigma \mathrm{a}^{2}+\sigma c^{2}+\right.$ $\left.\sigma \mathrm{e}^{2}\right)$. Thus, heritability $\left(h^{2}\right)$ in the narrow sense, which represents the proportion of residual variance attributed to additive genetic factors, is the ratio of the additive component and the total variance $\left(\sigma \mathrm{a}^{2} / \sigma \mathrm{p}^{2}\right)$. This assumes that all genetic variance is additive; in other words, there are no gene interactions.

\section{Results}

The demographic characteristics of the participants and the prevalence of an AN are presented in Table 1. The mean age of the participants was 43.99 years and the mean body mass index was 23.87. The prevalence of an $\mathrm{AN}$ in either foot was $40.96 \%$.

Table 2 shows the prevalence of an AN among participants in both feet and in both sexes. Type II was the most common type in both feet. There was no significant difference in the AN prevalence between right foot and left foot $(p>.05)$ and between both sexes $(p>.05)$.

Table 3 shows the laterality of an AN and the difference in the distribution of an AN subtypes between the right and left feet in bilateral cases. An AN tended to be present bilaterally $(69.36 \%)$ rather than in only one foot $(30.64 \%)$, and the majority $(85.16 \%)$ of bilateral ANs showed concordance between both sides. Among the bilaterally concordant cases, type II AN was more common than the other types. Among the bilaterally discordant cases, the type Itype II combination was the most common, followed by the II-III combination. 
TABLE 2

Prevalence (per 100 Persons) of Accessory Navicular Bone By Subtypes

\begin{tabular}{|c|c|c|c|c|c|c|}
\hline \multirow[b]{2}{*}{ Type } & \multicolumn{2}{|c|}{ All $(n=996)$} & \multicolumn{2}{|c|}{ Women $(n=604)$} & \multicolumn{2}{|c|}{ Men $(n=392)$} \\
\hline & Right foot & Left foot & Right foot & Left foot & Right foot & Left foot \\
\hline None & $664(66.67)$ & $637(63.96)$ & $391(64.74)$ & $382(63.25)$ & $272(69.64)$ & $255(65.05)$ \\
\hline Any type & $332(33.33)$ & $359(36.04)$ & $213(35.26)$ & $222(36.75)$ & $119(30.36)$ & $137(34.95)$ \\
\hline Type I & $127(12.75)$ & $116(11.65)$ & 89 (14.74) & $76(12.58)$ & 38 (9.69) & $40(10.20)$ \\
\hline Type II & $130(13.05)$ & $153(15.36)$ & $81(13.41)$ & $91(15.07)$ & $49(12.50)$ & $62(15.82)$ \\
\hline Type III & $75(7.53)$ & $90(9.04)$ & $43(7.12)$ & $55(9.11)$ & $32(8.16)$ & 35 (8.93) \\
\hline Agreement $[95 \% \mathrm{Cl}]^{*}$ & \multicolumn{2}{|c|}{$0.68[0.64,0.73]$} & \multicolumn{2}{|c|}{$0.67[0.62,0.73]$} & \multicolumn{2}{|c|}{$0.70[0.64,0.77]$} \\
\hline
\end{tabular}

Note: Data were presented as number (percentage).

*Agreement of accessary navicular bone on the right foot and left foot was evaluated by Cohen's kappa coefficient.

\section{TABLE 3}

Laterality of Accessory Navicular Bone and Side to Side Expression (Difference in the Distribution of Subtypes Between Right Foot and Left Foot) in Bilateral Cases

\begin{tabular}{|c|c|c|c|c|c|c|}
\hline \multirow[b]{2}{*}{ Side } & \multicolumn{2}{|c|}{ All $(n=996)$} & \multicolumn{2}{|c|}{ Women $(n=604)$} & \multicolumn{2}{|c|}{ Men $(n=392)$} \\
\hline & $N$ & $\%[95 \% \mathrm{Cl}]$ & $N$ & $\%[95 \% \mathrm{Cl}]$ & $N$ & $\%[95 \% \mathrm{Cl}]$ \\
\hline Bilateral & 283 & $28.41[25.61,31.21]^{a}$ & 178 & $29.47[25.83,33.11]^{a}$ & 105 & $26.79[22.41,31.17]^{a}$ \\
\hline Concordance & 241 & $85.16[81.02,89.30]^{b}$ & 149 & $83.71[78.29,89.13]^{b}$ & 92 & $87.62[81.32,93.92]^{\mathrm{b}}$ \\
\hline $1-1$ & 73 & $30.29[24.49,36.09]$ & 48 & $32.21[24.71,39.71]$ & 25 & $27.17[18.08,36.26]$ \\
\hline$\|-\|$ & 102 & $42.32[36.08,48.56]$ & 63 & $42.28[34.35,50.21]$ & 39 & $42.39[32.29,52.49]$ \\
\hline III-III & 66 & $27.39[21.76,33.02]$ & 38 & $25.50[18.50,32.50]$ & 28 & $30.43[21.03,39.83]$ \\
\hline Discordance & 42 & $14.84[10.70,18.98]^{b}$ & 29 & $16.29[10.87,21.71]^{b}$ & 13 & $12.38[6.08,18.68]^{b}$ \\
\hline $\mathrm{I}-\mathrm{II}$ & 25 & $59.52[44.67,74.37]$ & 19 & $65.52[48.22,82.82]$ & 6 & $46.15[19.05,73.25]$ \\
\hline$|-| I \mid$ & 3 & $7.14[-0.65,14.93]$ & 2 & $6.90[-2.32,16.12]$ & 1 & $7.69[-6.79,22.17]$ \\
\hline II-III & 14 & $33.33[19.07,47.59]$ & 8 & $27.59[11.32,43.86]$ & 6 & $46.15[19.05,73.25]$ \\
\hline Unilateral & 125 & $12.55[10.49,14.61]^{\mathrm{a}}$ & 79 & $13.08[10.39,15.77]^{a}$ & 46 & $11.73[8.54,14.92]^{a}$ \\
\hline
\end{tabular}

Note: a Percentage among total number of participants (996 in all, 604 in women, and 392 in men).

bPercentage among total number of bilateral accessory navicular bone (283 in all, 178 in women, and 105 in men).

Table 4 shows the intrapair agreement and heritability of AN. The intrapair agreement within MZ twin pairs was more than two times higher than that in other intrafamilial pairs, such as father-offspring pair, mother-offspring pair, and pooled DZ twin and sibling pairs. After adjusting for age and sex, the heritability estimate for the presence of any type of an AN in any foot was 0.88 (95\% CI [0.82, 0.94]). Specifically, type II AN showed the highest heritability of $0.82(95 \%$ CI $[0.71,0.93])$.

\section{Discussion}

In the present study of Korean healthy twins and their firstdegree family members, we found that an AN in the foot is a highly heritable trait. To date, there have been a few studies on the inheritance of an AN (Dobbs \& Walton, 2004; Kiter et al., 2000; Offenbecker \& Case, 2012).

Kiter et al. (2000) studied three generations of three pedigrees and found that an $\mathrm{AN}$ is transmitted in an autosomal dominant pattern with incomplete penetrance. Dobbs and Walton (2004) also verified the same result through studying two pedigrees of Scandinavian family. However, these studies stopped at unearthing the inheritance pattern because the number of participants was fewer than 80 . To the best of our knowledge, this is the first study to examine twins and their families to determine precise heritabil- ity of AN, with a large, sufficient number of participants of almost 1,000. Heritability estimation in a study involving both twins and their families may have a strength that overestimation of heritability can be avoided through the comparisons of phenotypic correlations between various familial relationships compared to the twin-only study or family-only study (Tenesa \& Haley, 2013).

In our study, among the different types of ANs, type II $\mathrm{AN}$ was the most common and the most highly heritable type among the different types. This is a clinically noteworthy finding as type II AN is also the most symptomatic type.

The laterality of an AN has been a lingering controversy. Some researchers have claimed the bilateral presence of an AN (Mygind, 1953; Shands \& Wentz, 1953), while another researcher claimed there is laterality in an AN (Coskun et al., 2009). In our study, there was a significant bilateral tendency of an AN and significant concordance in the type expression in both feet, with bilateral type II AN being the most common. These findings are strong evidence to support the bilateral tendency of AN.

Human height is a widely known inherited trait with an authorized prediction method for height of the next generation, the mid-parental height. Given that the reported heritability of height in humans is 0.8 (Silventoinen, 2003; Visscher et al., 2008) from a study by Silventoinen in 2003, and that the heritability of hallux valgus is 0.51 (Lee et al., 
TABLE 4

Intrafamilial Agreement* and Heritability of an Accessory Navicular Bone

\begin{tabular}{|c|c|c|c|c|c|}
\hline & \multicolumn{4}{|c|}{ Cohen's kappa coefficient $[95 \% \mathrm{Cl}]$} & \multirow[b]{2}{*}{$\begin{array}{l}\text { Heritability }\left(h^{2}\right) \\
\text { estimates }{ }^{\dagger} h^{2} \pm S E\end{array}$} \\
\hline & $\begin{array}{l}\text { MZ Twin pair } \\
\text { (135 pairs) }\end{array}$ & $\begin{array}{l}\text { Father-offspring } \\
\text { pair (280 pairs) }\end{array}$ & $\begin{array}{l}\text { Mother-offspring } \\
\text { pair (477 pairs) }\end{array}$ & $\begin{array}{l}\text { Pooled DZ and SB } \\
\text { pairs (266 pairs) }\end{array}$ & \\
\hline \multicolumn{6}{|l|}{ Right foot } \\
\hline Any type & $0.53[0.37,0.69]$ & $0.14[0.03,0.25]$ & $0.24[0.16,0.33]$ & $0.32[0.20,0.43]$ & $0.73 \pm 0.13$ \\
\hline Type I & $0.41[0.18,0.64]$ & $0.06[-0.07,0.20]$ & $0.02[-0.07,0.11]$ & $0.01[-0.11,0.13]$ & $0.35 \pm 0.07$ \\
\hline Type II & $0.74[0.53,0.96]$ & $0.06[-0.07,0.18]$ & $0.10[0.01,0.20]$ & $0.20[0.05,0.36]$ & $0.82 \pm 0.11$ \\
\hline Type III & $0.60[0.34,0.87]$ & $0.13[-0.04,0.29]$ & $0.13[-0.00,0.27]$ & $-0.01[-0.12,0.10]$ & $0.72 \pm 014$ \\
\hline Overall\$ & $0.69[0.57,0.82]$ & $0.25[0.14,0.35]$ & $0.25[0.16,0.33]$ & $0.26[0.15,0.38]$ & $0.88 \pm 0.06$ \\
\hline
\end{tabular}

Note: $\mathrm{MZ}=$ monozygotic twins, $\mathrm{DZ}=$ dizygotic twins, $\mathrm{SB}=$ sibling, $\mathrm{SE}=$ standard error

*Assessed by Cohen's kappa coefficient. ${ }^{\dagger}$ Adjusted for age and and sex. ${ }^{\S}$ Any type of an accessory navicular bone in the right foot or the left foot.

2014), the heritability estimate of 0.88 implies a very strong genetic contribution to the presence of an $\mathrm{AN}$ in the foot. As the clinical role of an $\mathrm{AN}$ in foot pathology is considerable due to its association with posterior tibial tendon insufficiency, malalignment of the medial foot arch and probably pes planus, high heritability of an AN needs to be further emphasized. Being well acquainted with high heritability and bilateral tendency of an AN would aid clinically in dealing with foot pain in patients with a family history of an AN. The physicians should be aware of the possibility of an AN in order to avoid missing or misdiagnosing a symptomatic $\mathrm{AN}$ as simple arthritis or any other disease pathology.

The present study has a limitation to be considered. First, the presence and the type of an AN were ascertained using foot X-rays with only the antero-posterior and lateral weight-bearing views. An additional lateral oblique view of foot X-ray is often helpful in determining the subtypes of type II and III ANs, and occasionally for differentiation of type II AN from type III AN in difficult cases. Therefore, we think an extra oblique view would have improved the precision of radiographic assessment, especially regarding the type differentiation. However, the effect of the limited X-ray view seems to have had a modest impact on the findings of our study, given that the presence of an $\mathrm{AN}$ can still be determined simply by an antero-posterior view (Vaughan \& Singh, 2014). Moreover, the high interrater reliability (kappa coefficients ranged between 0.74 and 0.95 ) between the two measurers of an AN in our study also supports that significant measurement bias is less likely to be involved. Second, we estimated narrow sense heritability of AN. Given that the narrow sense heritability assumes all genetic variance as additive and does not consider dominance variance or epistasis, the heritability estimation of an AN in our study might have been a bit inflated.

In conclusion, we found that genetic influence plays a major role in the development of an $\mathrm{AN}$ in the foot, using the data of Korean healthy twins and their first degree family members. This finding may serve an evidence supporting that prediction of an $\mathrm{AN}$ in a person is possible by reviewing a family history of AN. Further studies are needed to elucidate genes that are involved in determination of an AN development.

\section{Acknowledgments}

This research was supported by the Basic Science Research Program through the National Research Foundation of Korea(NRF) funded by the Ministry of Science, ICT and future Planning(2014R1A2A2A01002705).

\section{Disclosure of Interests}

None.

\section{Supplementary material}

To view supplementary material for this article, please visit https://doi.org/10.1017/thg.2017.21

\section{References}

Allison, D. B., Neale, M. C., Zannolli, R., Schork, N. J., Amos, C. I., \& Blangero, J. (1999). Testing the robustness of the likelihood-ratio test in a variance-component quantitativetrait loci-mapping procedure. American Journal of Human Genetics, 65, 531-544.

Almasy, L., \& Blangero, J. (1998). Multipoint quantitative-trait linkage analysis in general pedigrees. American Journal of Human Genetics, 62, 1198-1211.

Bernaerts, A., Vanhoenacker, F. M., Van De Perre, S., De Schepper, A. M., \& Parizel, P. M. (2004). Accessory navicular bone: Not such a normal variant. Journal Belge de Radiologie - Belgisch Tijdschrift voor Radiologi, 87, 250-252.

Chen, Y. J., Hsu, R. W., \& Liang, S. C. (1997). Degeneration of the accessory navicular synchondrosis presenting as rupture of the posterior tibial tendon. Journal of Bone and Joint Surgery, 79, 1791-1798.

Coskun, N., Yuksel, M., Cevener, M., Arican, R. Y., Ozdemir, H., Bircan, O. ... Sindel, M. (2009). Incidence of accessory ossicles and sesamoid bones in the feet: A radiographic study of the Turkish subjects. Surgical and Radiologic Anatomy, 31, 19-24.

Coughlin, M. J. (2006). Sesamoid and accessory bones of the foot: Surgery of the foot and ankle. Amsterdam: Elsevier.

Dobbs, M. B., \& Walton, T. (2004). Autosomal dominant transmission of accessory navicular. Iowa Orthopaedic Journal, 24, 84-85. 
Gombojav, B., Song, Y. M., Lee, K., Yang, S., Kho, M., Hwang, Y. C., ... Sung, J. (2013). The healthy twin study, Korea updates: Resources for omics and genome epidemiology studies. Twin Research and Human Genetics, 16, 241-245.

Huang, J., Zhang, Y., Ma, X., Wang, X., Zhang, C., \& Chen, L. (2014). Accessory navicular bone incidence in Chinese patients: A retrospective analysis of X-rays following trauma or progressive pain onset. Surgical and Radiologic Anatomy, 36, 167-172.

Keles Coskun, N., Arican, R. Y., Utuk, A., Ozcanli, H., \& Sindel, T. (2009). The incidence of accessory navicular bone types in Turkish subjects. Surgical and Radiologic Anatomy, 31, 675-679.

Kiter, E., Erdag, N., Karatosun, V., \& Gunal, I. (1999). Tibialis posterior tendon abnormalities in feet with accessory navicular bone and flatfoot. Acta Orthopaedica Scandinavica, $70,618-621$.

Kiter, E., Erduran, M., \& Gunal, I. (2000). Inheritance of the accessory navicular bone. Archives of Orthopaedic and Trauma Surgery, 120, 582-583.

Lee, C. H., Lee, S., Kang, H., Jung, D. E., Song, Y. M., Lee, K., ... Sung, J. (2014). Genetic influences on hallux valgus in Koreans: The healthy twin study. Twin Research and Human Genetics, 17, 121-126.

Mikami, M., \& Azuma, H. (1978). Fracture of the os tibiale externum. A case report. Journal of Bone and Joint Surgery, 60, 556-557.

Miller, T. T., Staron, R. B., Feldman, F., Parisien, M., Glucksman, W. J., \& Gandolfo, L. H. (1995). The symptomatic accessory tarsal navicular bone: Assessment with MR imaging. Radiology, 195, 849-853.
Mygind, H. B. (1953). The accessory tarsal scaphoid; clinical features and treatment. Acta Orthopaedica Scandinavica, $23,142-151$.

Offenbecker, A. M., \& Case, D. T. (2012). Accessory navicular: A heritable accessory bone of the human foot. International Journal of Osteoarchaeology, 22, 158-167.

Seehausen, D. A., Harris, L. R., Kay, R. M., \& Pace, J. L. (2016). Accessory navicular is associated with wider and more prominent navicular bone in pediatric patients by radiographic measurement. Journal of Pediatric Orthopaedics, 36, 521-525.

Shands, A. R. Jr., \& Wentz, I. J. (1953). Congenital anomalies, accessory bones, and osteochondritis in the feet of 850 children. Surgical Clinics of North America, 1643-1666.

Silventoinen, K. (2003). Determinants of variation in adult body height. Journal of Biosocial Science, 35, 263285.

Song, Y. M., Lee, D., Lee, M. K., Lee, K., Lee, H. J., Hong, E. J., ... Sung, J. (2010). Validity of the zygosity questionnaire and characteristics of zygosity-misdiagnosed twin pairs in the Healthy Twin Study of Korea. Twin Research and Human Genetics, 13, 223-230.

Tenesa, A., \& Haley, C. S. (2013). The heritability of human disease: Estimation, uses and abuses. Nature Reviews: Genetics, 14, 139-149.

Vaughan, P., \& Singh, D. (2014). Ongoing pain and deformity after an excision of the accessory navicular. Foot and Ankle Clinics, 19, 541-553.

Visscher, P. M., Hill, W. G., \& Wray, N. R. (2008). Heritability in the genomics era-concepts and misconceptions. Nature Reviews Genetics, 9, 255-266. 